\title{
Acute phlegmonous gastritis
}

\author{
P.A. O'Toole ${ }^{1}$ and J.A. Morris ${ }^{2}$ \\ ${ }^{1}$ Department of Medicine, Royal Lancaster Infirmary, Lancaster LA1 4RP and ${ }^{2}$ Lancaster Moor Hospital, \\ Lancaster LA1 3JR, UK.
}

\begin{abstract}
Summary: A fatal case of acute phlegmonous gastritis in a $\mathbf{3 0}$ year old man is reported. It was caused by Group A Streptococcus (M type 1, T type 1). Although extremely rare, clinicians need to be aware of this condition as, without intervention, it is rapidly fatal. The recommended treatment is antibiotics combined with surgical resection.
\end{abstract}

\section{Introduction}

Acute phlegmonous gastritis is an extremely uncommon rapidly progressive bacterial infection which is rarely diagnosed or even considered until laparotomy or autopsy. It might have been more common in the past, however, as it was known to Galen, described by Cruveilhier ${ }^{1}$ in 1862 , and by 1919 Sundberg $^{2}$ was able to review 215 cases. Since 1919 single case reports have continued at approximately one per year, although many have been in the East European literature. The continued use of the somewhat archaic term phlegmonous, which means a diffuse spreading inflammation of connective tissue, reflects this long history and its current rarity. However, the condition is important and practitioners need to be aware of it if a fatal outcome is to be avoided.

\section{Case report}

A 30 year old unmarried man presented with upper abdominal pain of 3 days duration. It was associated with nausea, occasional vomiting and marked belching. His average daily alcohol intake was $80 \mathrm{~g}$. On examination he was apyrexial $\left(37.2^{\circ} \mathrm{C}\right)$, mildly dehydrated, tachypnoeic and had a pulse rate of 120 /minute with a blood pressure of $100 / 60 \mathrm{mmHg}$. There was tenderness in the upper abdomen but no clinical evidence of peritonitis. A plain abdominal $\mathrm{X}$-ray was normal. The serum sodium was $119 \mathrm{mmol} / \mathrm{l}$, urea $134 \mu \mathrm{mol} / 1$, albumin $22 \mathrm{~g} / \mathrm{l}$, glucose $18.7 \mathrm{mmol} / 1$, amylase $101 \mathrm{U} / 1$, haemoglobin $14.1 \mathrm{~g} / \mathrm{dl}$, and white cell count $12.9 \times 10^{9} / 1$. His urine contained protein and glucose but was negative for ketones. There was no previous medical

Correspondence: J.A. Morris, M.A., M.R.C.Path.

Accepted: 25 November 1987 history of diabetes mellitus, but in view of the hyperglycaemia he was treated with intravenous fluids and insulin. However he suffered a cardiac arrest 12 hours after admission and attempts at resuscitation were unsuccessful. A blood culture taken prior to death subsequently grew Group A Streptococcus.

Post-mortem examination revealed a small amount of turbid fluid in the peritoneal cavity with a mild inflammatory reaction on the intestinal serosal surface. The stomach was distended with a purulent serosal exudate and gross inflammatory thickening of the wall. On section the wall exuded pus and there was haemorrhagic necrosis of the submucosa. These changes terminated abruptly at the pylorus and the gastro-oesophageal junction. Histological examination revealed severe transmural inflammation of the stomach with a heavy infiltrate of neutrophils and many Gram positive cocci in chains. There was no significant macroscopic or histological abnormality of the other body organs. In particular the pancreas was normal, and there was no histological evidence of previous or current damage to the islets of Langerhans. Group A Streptococci (M type 1, T type 1) were grown from the blood, peritoneal fluid and stomach wall.

\section{Discussion}

The post-mortem diagnosis was acute phlegmonous gastritis with streptococcal septicaemia. There was no histological evidence at autopsy to support a diagnosis of diabetes mellitus and the hyperglycaemia was presumably a stress response to the systemic illness. A normal body temperature on admission, in the presence of systemic streptococcal infection, was another unusual feature of this case.

(C) The Fellowship of Postgraduate Medicine, 1988 
Acute phlegmonous gastritis affects both sexes and occurs most commonly in the 30 to 70 age range. ${ }^{3,4}$ Chronic gastritis, chronic alcoholism and general debility are predisposing factors although, as in this case, it can affect those who were previously well. The majority of cases have been caused by haemolytic streptococci, although pneumococci, staphylococci, Escherichia coli, Proteus, Haemophilus influenzae and Clostridia have been responsible for some cases. ${ }^{3.4}$ It is not known by which route the infection is acquired. One possibility is direct spread from the gastric lumen due to the ingestion of infected respiratory tract secretions, in which case acute or chronic gastritis could act by reducing acid secretion and providing a portal of entry through the damaged mucosa. A second possible route of infection is blood-borne spread to the stomach from a distant focus.

The condition presents with a severe systemic illness associated with epigastric pain and tenderness which is often relieved by sitting forward. ${ }^{3,4}$ The diagnosis can be suspected but is rarely made pre-mortem without laparotomy. However it has recently been claimed that ultrasound

\section{References}

1. Cruveilhier, J. Traite d'Anatomie Pathologique Generale, vol. 4. Masson et Cie, Paris, 1862, p 485.

2. Sundberg, H.H. Ueber gastric phlegmone. Nord Med Arkiv 1919, II: 303-468.

3. Starr, A. \& Wilson, J.M. Phlegmonous gastritis. Ann Surg 1957, 145: 88-93.

4. Stephenson, S.E., Yasrebi, H., Rhatigan, R. \& Woodward, E.R. Acute phlegmasia of the stomach. Am Surg 1970, 36: 225-231.

5. Lifton, L.J. \& Schlossberg, D. Phlegmonous gastritis after endoscopic polypectomy. Ann Intern Med 1982, 97: 373-374. examination of the abdomen shows a characteristic picture with diffuse thickening of the gastric wall and luminal dilatation..$^{5,6}$ The disease has also been diagnosed at endoscopy ${ }^{5,7}$ and by bacterial examination of gastric secretions. ${ }^{8}$ In the preantibiotic era it was almost always fatal. Miller et al. reviewed 25 cases reported since $1945^{\circ}$ and found there was still $100 \%$ mortality in those treated medically although this was reduced to $18 \%$ when surgical resection was combined with antibiotics. Thus the recommended treatment is antibiotics and resection of the involved area.

Acute phlegmonous gastritis receives scant attention in standard textbooks of medicine and surgery and most medical practitioners are unaware of its existence. The last few years, however, have seen a rising incidence of serious streptococcal infection in the USA and Great Britain (PHLS, Colindale, personal communication), and it is possible that acute phlegmonous gastritis will be more common in the future. If so doctors need to be aware of it and we hope that this report contributes to that awareness.

6. Tierney, L.M., Gooding, G., Bottles, K., Montgomery, C.K. \& Fitzgerald, F.T. Phlegmonous gastritis and Haemophilus influenzae peritonitis in a patient with alcoholic liver disease. Dig Dis Sci 1987, 32: 97-101.

7. Bron. B.A., Deyhle, P., Pelloni, S., Krejs, G.J., Siebenmann, R.E. \& Blum, A.L. Phlegmonous gastritis diagnosed by endoscopic snare biopsy. Am J Dig Dis 1977, 22: 729-733.

8. Marshall, C.J. Phlegmonous gastritis. Ann Surg 1927, 85: 668-682.

9. Miller, A.I., Smith, B. \& Rogers, A.I. Phlegmonous gastritis. Gastroenterology 1975, 68: 231-238. 